\title{
Perinatal outcomes in pregnant women presenting with preterm premature rupture of membranes at a regional hospital in KwaZulu- Natal Province, South Africa
}

\author{
G Msomi, ${ }^{1}$ MB ChB; M Naidoo, ${ }^{2}$ MB ChB, MFamMed, FCFP, MSc (Sports Med), PhD; B Hira, ${ }^{1}$ MB BCh, FCOG \\ ${ }^{1}$ Discipline of Obstetrics and Gynaecology, School of Clinical Medicine, University of KwaZulu-Natal, Durban, South Africa \\ ${ }^{2}$ Discipline of Family Medicine, School of Nursing and Public Health, University of KwaZulu-Natal, Durban, South Africa
}

Corresponding author: M Naidoo (naidoom@ukzn.ac.za)

\begin{abstract}
Background. Worldwide, the incidence of preterm premature rupture of membranes (PPROM) is between $1 \%$ and $4 \%$ of all pregnancies. Objectives. The primary objectives of this study were to describe and compare the perinatal outcomes of HIV-positive and HIV-negative women presenting with PPROM to a regional hospital in KwaZulu-Natal.

Methods. This was a retrospective analytical cross-sectional study which reviewed files of pregnant women presenting with premature rupture of membranes at gestation between 28 and 36 completed weeks. These were identified from the labour ward birth register and from the neonatal ward admission book. Categorical and numerical variables pertaining to the method of confirmation of diagnoses, clinical profiles, modes of delivery, maternal outcomes and neonatal outcomes were considered.

Results. A total of 87 files were analysed. Forty-six women (53\%) were HIV-negative and 41 (47\%) were HIV-positive. Fifty-two percent were in the gestational age $<34$ weeks. Fifty-nine percent $(n=51)$ of women delivered vaginally and $31 \%(n=27)$ delivered by caesarean delivery (CD). There was also no statistical significance between the Apgar scores of the HIV-exposed and HIV-unexposed neonates, birth weights and modes of delivery. There was no statistical significance in sepsis rates, the need for ventilation and the duration of hospital stay between the two groups. The odds of developing neonatal jaundice (NNJ) in the HIV-positive group was 0.14 (95\% confidence interval (CI) 0 - 0.93), which was statistically significant. There was no reported maternal or neonatal mortality and no maternal morbidity associated with PPROM in either groups.

Conclusion. This study suggests that there are no immediate significant differences in neonatal and maternal outcomes in pregnancies complicated by PPROM between HIV-negative and HIV-positive women on ART except that of NNJ.
\end{abstract}

S Afr J Obstet Gynaecol 2017;23(2):43-47. DOI:10.7196/SAJOG.2017.v23i2.1124

Preterm delivery and prematurity are significant contributors to perinatal morbidity and mortality, and their inclusion in the list of sustainable development goal epitomises this. Worldwide, the incidence of preterm delivery is between $1 \%$ and $4 \%$ of all pregnancies, with higher incidence notably in low-income countries. ${ }^{[1]}$ This figure rises significantly if a patient has a history of premature rupture of membranes (PROM). It is estimated that $30-40 \%$ of cases of preterm delivery are preceded by PROM..$^{[2-6]}$ As a complication of pregnancy, PROM has potentially devastating perinatal and maternal outcomes, not to mention the management dilemma it poses to the obstetrician. It is therefore no surprise that much international research has been done on this subject, with varying and sometimes conflicting findings.

The role of HIV as a confounding factor to preterm PROM (PPROM) has, however, not yet been adequately described. There are no clear distinctions in the management of PPROM in HIV-positive and HIV-negative groups. Studies have found an increased incidence of preterm deliveries, low birth weights and perinatal deaths as the main associated complications in the HIV-positive group. ${ }^{[7-11]}$ Other disputed complications in this group include increased risk of intrapartum hypoxia and neonatal encephalopathy. ${ }^{[12-15]}$

HIV and chorioamnionitis have mutually deleterious effects on PPROM, as HIV is implicated in the pathogenesis of chorioamnionitis, and HIV-positive patients who develop PPROM are much more likely to develop chorioamnionitis. ${ }^{[16]}$ The presence of chorioamnionitis, with or without rupture of membranes, can in turn increase the risk of vertical transmission of the virus from mother to the fetus. ${ }^{[16,17]}$

Vertical transmission of the virus from mother to child has been researched in great depth, with encouraging results regarding the use of antiretroviral treatment (ART) in reducing transmission. The risk of vertical transmission varies based on the stages of pregnancy, with the highest risk occurring during parturition. ${ }^{[18]}$ Increases in the duration of the rupture of membranes, prematurity and prolonged labour after rupture of membranes increase the risk of perinatal HIV transmission. ${ }^{[3,18]}$ Some studies have reported an increased risk of preterm deliveries with the use of antiretroviral drugs (ARVs). ${ }^{[20,21]}$

It is against this background that this study investigated the perinatal outcomes of HIV-positive, compared with HIV-negative women presenting with PPROM at the regional hospital in KwaZulu-Natal Province (KZN), South Africa.

\section{Methods}

We retrospectively analysed the files of pregnant women who presented with PPROM to a regional hospital in KZN from 1 January 2013 to 31 of December 2013. This hospital offers a regional level of 
care to the surrounding suburban population. It also serves as a referral point for a nearby district hospital, rural and urban primary healthcare clinics, and serves a population of $\sim 400000$ people. Due to the limitations of our neonatal facilities, viability is restricted to gestation of 28 weeks or a birth weight of $1000 \mathrm{~g}$.

Inclusion criteria were clinical files of women who presented or were referred with a history of having ruptured membranes, without exhibiting any signs of labour, and were between the 28th and 37th week of gestation. In the management of a fully developed fetus with PROM decision-making is generally less complicated. Conversely, the management of pregnancies of gestation less than 28 weeks is far more complex and inconsistent, thereby making it difficult to study retrospectively. Women with existing medical conditions such as hypertensive diseases that could influence perinatal outcomes were excluded. Further exclusion criteria were unknown gestational age, unknown HIV-status, congenital abnormalities and cases where corresponding neonatal files were missing.

When reviewing the clinical files, rupture of membranes was confirmed by either visualisation of amniotic fluid in the vagina on speculum examination, a convincing history of drainage of amniotic fluid, plus reduced amniotic fluid index on ultrasound scan or by observing persistent amniotic fluid staining of sanitary pads. The corresponding neonatal charts were also reviewed. It is important to note that, regardless of the gestational age, this institution does not offer expectant management for women with PPROM.

A data collection sheet was used to extract the required information from the medical files. Maternal and obstetric characteristics included demographic data, gestation, number of previous pregnancies and relevant obstetric history, HIV-status, rhesus factor and syphilis serology. Clinical data were assessed in respect of relevant clinical findings: laboratory investigations (haematology and microbiology), ultrasound scans, administration of antibiotics and corticosteroids, induction of labour and the eventual mode of delivery.

The primary outcomes of this study were the perinatal and maternal morbidity and mortality occurring before discharge. Collated perinatal data included Apgar scores at 1 and 5 minutes, admission to the neonatal ward or intensive care unit, need for ventilation, development of any of the following conditions: hyaline membrane disease, hypoxic ischaemic encephalopathy, intraventricular haemorrhage, congenital or nosocomial infections, necrotising enterocolitis and death. Maternal morbidity included increased length of hospital stay (more than 12 hours following vaginal delivery and more than 72 hours following caesarean section (CS)).

The variables on the data sheet were captured on a Microsoft Excel spreadsheet and imported into Stata version 13 (StataCorp LP, USA) for analysis. The HIV-positive and HIV-negative subgroups were then compared. The statistical analysis was performed using Pearson $\chi^{2}$ test with $p<0.05$ considered significant. We determined the mean and odds ratios (OR) for various outcomes between the two cohorts and their $95 \%$ confidence intervals (CI) were calculated. The study was approved by the Biomedical Ethics Committee at the University of KwaZulu-Natal (ref. no. BE 275/14), the provincial Department of Health and the facility manager.

\section{Results}

There was a total of 7694 deliveries in 2013. Out of a total of 130 $(0.2 \%)$ files of patients who potentially met the inclusion criteria only 87 files were included in this study. Forty-three files were either missing or lacked the crucial information required for the study. Most women $(n=64 ; 74 \%)$ fell in the age bracket of 18 - 34 years, with $13(15 \%)$ women $<18$ years, and $(n=10 ; 11 \%)>34$ years of age.

Forty-six women (53\%) were HIV-negative and 41 (47\%) were HIV-positive. In the HIV-positive cohort 5 (12\%) patients had CD4 counts $<200$ cells/ $\mu \mathrm{L}, 13$ (32\%) between 200 and 350 cells $/ \mu \mathrm{L}$, $20(49 \%)>500$ cells/ $\mu \mathrm{L}$, and $3(7 \%)$ patients had unknown CD4 counts. All HIV-positive women were on a triple ART regimen. The clinical information of all reviewed patients is presented in Table 1.

Regarding the patients' gestational age, $24 \%(n=21)$ were between 28 and 32 weeks, $28 \%(n=24)$ were between 32 and 34 weeks, while $48 \%(n=42)$ were between 34 and 36 weeks. There were no statistical

Table 1. Comparison of clinical information of HIV-positive $(n=41)$ and HIV-negative $(n=46)$ participants

\begin{tabular}{|c|c|c|c|}
\hline & $\begin{array}{l}\text { HIV- } \\
\text { negative, } \\
n(\%)\end{array}$ & $\begin{array}{l}\text { HIV- } \\
\text { positive, } \\
n(\%)\end{array}$ & $p$-value \\
\hline \multicolumn{4}{|l|}{ Gestation (weeks) } \\
\hline $28-32$ & $9(20)$ & $12(29)$ & 0.21 \\
\hline $33-34$ & $13(28)$ & $11(27)$ & 0.53 \\
\hline $35-36$ & $24(52)$ & $18(44)$ & 0.29 \\
\hline \multicolumn{4}{|c|}{$\begin{array}{l}\text { Time from PPROM to delivery } \\
\text { (hours) }\end{array}$} \\
\hline$<12$ & 0 & $3(7)$ & 0.10 \\
\hline$>12$ and $<24$ & $10(22)$ & $8(19)$ & 0.51 \\
\hline$>24$ & $30(65)$ & $24(59)$ & 0.54 \\
\hline Unknown & $6(13)$ & $6(15)$ & \\
\hline \multicolumn{4}{|l|}{ Confirmation of SROM } \\
\hline Speculum & $20(43)$ & $18(44)$ & 0.57 \\
\hline Pad inspection & $5(11)$ & $9(22)$ & 0.13 \\
\hline History and low AFI & $4(9)$ & $3(7)$ & 0.57 \\
\hline Digital exam & $6(13)$ & $9(22)$ & 0.21 \\
\hline Unknown & $11(24)$ & $2(5)$ & 0.01 \\
\hline \multicolumn{4}{|l|}{ Tocolysis } \\
\hline Yes & $5(11)$ & $9(22)$ & 0.13 \\
\hline No & $35(76)$ & $29(71)$ & 0.37 \\
\hline Unknown & $6(13)$ & $3(7)$ & 0.30 \\
\hline \multicolumn{4}{|l|}{ Steroid administration } \\
\hline Yes & $14(30)$ & $21(51)$ & 0.38 \\
\hline No & $27(59)$ & $18(44)$ & 0.12 \\
\hline Unknown & $5(11)$ & $2(5)$ & 0.27 \\
\hline \multicolumn{4}{|l|}{ Mode of delivery } \\
\hline NVD & $30(65)$ & $28(68)$ & 0.47 \\
\hline $\mathrm{CD}$ & $16(35)$ & $13(32)$ & 0.47 \\
\hline \multicolumn{4}{|l|}{ Induction of labour } \\
\hline Yes & $11(24)$ & $11(27)$ & 0.54 \\
\hline No & $14(30)$ & $13(32)$ & 0.43 \\
\hline Unknown & $21(46)$ & $17(41)$ & \\
\hline $\begin{array}{l}\mathrm{SROM}=\text { spontaneous rupture o } \\
\mathrm{CD}=\text { caesarean delivery; } \mathrm{AFI}= \\
\text { of membranes. }\end{array}$ & $\begin{array}{l}\mathrm{NVD}=\text { no } \\
1 \text { index: } \mathrm{PPP}\end{array}$ & vaginal deli & ture ruptur \\
\hline
\end{tabular}


differences pertaining to the clinical profile and management of the HIV-positive and HIV-negative women, except that women who were HIV-positive had steroids administered significantly more often.

The mean number of antenatal visits in the HIV-negative group was 4.17 (95\% CI 3.43 - 4.91) whereas in the HIV-positive group it was 4.31 (95\% CI 3.79 - 4.84) and there was no statistically significant difference between the 2 groups. Only 2 patients had no antenatal visits and both were HIV-negative. The number of patients who booked before 20 weeks of gestation in the HIV-negative and HIV-positive groups were 15 (33\%) and 16 (39\%), respectively, and this was not statistically significant. All the booked patients in this study were rhesus-positive. Only one patient was HIV-positive and had a positive screening test for syphilis.

In 38 (44\%) women, the drainage of amniotic fluid was confirmed with the recommended use of a speculum. Ultrasound amniotic fluid index and regular pad inspection confirmed rupture of membranes in $7(8 \%)$ and $14(16 \%)$ women, respectively. Notably, $15(17 \%)$ had the rupture of membranes confirmed by the observation of leakage of amniotic fluid during digital per vaginam examination. The common bacteriological risk factors for premature rupture of membranes could not be determined in both groups, as very few patients had specimens sent for analysis. One HIV-negative patient had a Bartholin's abscess and one HIV-positive patient had a urinary tract infection. None of the women exhibited any other pregnancy-related infections.

A total of 21 women $(24 \%)$ had a latency period from rupture of membranes to delivery of $<24$ hours; $10(22 \%)$ were in the HIV-negative

Table 2. Comparison of neonatal outcomes of HIV-negative $(N=46)$ and HIV-positive $(N=41)$ women

\begin{tabular}{|c|c|c|c|}
\hline & $\begin{array}{l}\text { HIV- } \\
\text { negative, } \\
n(\%)^{*}\end{array}$ & $\begin{array}{l}\text { HIV- } \\
\text { positive, } \\
n(\%)^{\star}\end{array}$ & $p$-value \\
\hline \multicolumn{4}{|c|}{ Birth weight (grams) } \\
\hline $1000-1499$ & $4(9)$ & $7(17)$ & 0.20 \\
\hline $1500-1999$ & $9(20)$ & $12(29)$ & 0.21 \\
\hline $2000-2499$ & $27(59)$ & $16(39)$ & 0.05 \\
\hline$>2500$ & $6(13)$ & $6(15)$ & 0.30 \\
\hline \multicolumn{4}{|l|}{ Sex } \\
\hline Male & $18(39)$ & $25(61)$ & 0.03 \\
\hline Female & $28(61)$ & $16(39)$ & 0.03 \\
\hline \multicolumn{4}{|l|}{ Apgar at $5 \mathrm{~min}$} \\
\hline $8-10$ & $45(98)$ & $39(95)$ & 0.64 \\
\hline$<8$ & $1(2)$ & $2(5)$ & 0.64 \\
\hline \multicolumn{4}{|c|}{ Time spent in nursery (hours) } \\
\hline$<24$ & $14(30)$ & $12(15)$ & 0.55 \\
\hline $24-48$ & $2(4)$ & $7(8)$ & 0.06 \\
\hline$>48$ & $8(17)$ & $11(14)$ & 0.21 \\
\hline $\begin{array}{l}\text { Number needing } \\
\text { ventilation }\end{array}$ & $2(4)$ & $2(5)$ & 0.64 \\
\hline \multicolumn{4}{|c|}{$\begin{array}{l}\text { Length of hospital stay post delivery } \\
\text { (days) }\end{array}$} \\
\hline$<3$ & $22(48)$ & $21(51)$ & 0.46 \\
\hline $3-7$ & $19(41)$ & $13(32)$ & 0.40 \\
\hline$>7$ & $5(11)$ & $7(17)$ & 0.30 \\
\hline
\end{tabular}

group and 11 (27\%) were in the HIV-positive group. The difference was not statistically significant. Three women delivered within 12 hours and all were in the HIV-positive group. The majority of women (62\%) had a latency of $>24$ hours, 30 (65\%) and 24 (56\%) in the HIV-negative and HIV-positive groups, respectively.

Women who had ruptured membranes for $>12$ hours and those who warranted suppression of labour to facilitate steroid lung maturity were routinely given prophylactic antibiotics. Fifty-seven (66\%) women were given oral erythromycin and metronidazole in accordance with the hospital management protocol. Four women who were eligible for antibiotics were not prescribed any and 5 had antibiotics mentioned in the management plan, but no prescriptions were found in the files. Eight women were already in labour when the 12-hour period had elapsed. Antibiotic treatment was not prescribed in 9 patients.

Fifteen women (16\%) had their labour suppressed using nifedipine to allow for fetal lung maturity. Betamethasone was given to 35 of the 45 women eligible to receive steroids for fetal lung maturity. There were no statistically significant differences between the 2 groups with respect to tocolysis and the use of steroids.

Twenty-two (25\%) women had induced labour; 51 (59\%) delivered vaginally and 27 (31\%) delivered by caesarean delivery (CD). Indications for $\mathrm{CD}$ included fetal distress $(n=7)$, breech presentation $(n=5)$, anhydramnios $(n=3)$, failed induction of labour $(n=3)$, previous $\mathrm{CD} \times 1 \quad(n=4)$, previous $\mathrm{CD} \times 2(n=2)$, poor progress of labour $(n=2)$, cord prolapse $(n=1)$, high parity $(n=1)$ and very low fetal weight $(n=1)$. There were no statistically significant differences in the induction of labour and mode of delivery between the HIVnegative and HIV-positive groups.

\section{Maternal outcomes}

None of the mothers suffered any intrapartum or postpartum complications until discharge. Mothers who stayed in hospital for $>3$ days were kept as boarders awaiting completion of the intravenous antibiotic course for their babies and/or for adequate neonatal weight gain.

\section{Neonatal outcomes}

In the baby-weight category, 43 (49\%) babies weighed 2000 - $2500 \mathrm{~g}$, 21 (24\%) weighed $1500-1999 \mathrm{~g}, 12$ (14\%) weighed >2 $500 \mathrm{~g}$ and $11(13 \%)$ weighed $1000-1500 \mathrm{~g}$. There were significantly more males in the HIV-positive group and more females in the HIVnegative group $(p=0.03)$. Clinical information on neonatal outcomes is presented in Table 2.

Overall, 54 babies were admitted to the nursery, 24 were HIV-negative and 30 were HIV-positive. Invasive ventilation was administered to 2 babies in each group. Twenty-six (30\%) were discharged from the nursery within 24 hours, 9 (10\%) were discharged between 24 and 48 hours and 19 (23\%) were in the nursery for $>48$ hours.

The odds of being admitted to the nursery for $>48$ hours in the HIV-positive group was 1.74 (95\% CI 0.64 - 4.76) and the odds of staying in hospital for more than 7 days in the HIV-positive infants was 1.68 (95\% CI $0.51-5.51)$. Both odds ratios were not statistically significant. There were no correlations between CD4 cell counts of the mothers and the length of their stays in either the nursery or the hospital.

Twenty-three (26\%) babies received intravenous antibiotics for suspected congenital sepsis on the basis of a raised white cell count. Twelve (26\%) were in the HIV-negative group and 7 (17\%) were HIV-positive. This finding was not statistically significant 
( $p=0.44)$. Routine neonatal septic screening by means of $\mathrm{C}$ reactive protein (CRP) and blood culture investigations were not helpful in confirming infection, except in 2 babies, where contamination was suspected.

A total of 8 babies (9\%) developed neonatal jaundice (NNJ). Of these, only 1 was HIV-negative; 3 were male and 5 were female. The odds of developing NNJ in the HIV-positive group was 0.14 (95\% CI 0 - 0.93), which was statistically significant. The mean birth weight was $2450 \mathrm{~g}$, with a range of $1200-2900 \mathrm{~g}$. Six women delivered vaginally, while 2 delivered by $C D$. No babies suffered neurological or gastrointestinal complications associated with PPROM before discharge. There was also no perinatal mortality in the analysed files.

\section{Discussion}

This retrospective study found no significant differences in the perinatal and maternal outcomes of HIV-positive and HIV-negative women with pregnancies complicated by PPROM. There were no differences in respect of respiratory complications, neonatal intensive care unit admission, neonatal infections and length of hospital stay post delivery. None of the babies developed any neurological or gastrointestinal complications until they were discharged and there was surprisingly no perinatal mortality. This study also concurs with previous studies performed in other African countries, comparing HIV-positive and HIV-negative cohorts, which found little or no differences between HIV-positive and HIV-negative women with respect to gestational age, birth weight, and Apgar scores. ${ }^{[22,23]}$

We could find no explanation for the skewed distribution of the sexes among the 2 groups. The differences were statistically significant. This could be a statistical error due to the higher number of missing or disqualified files.

Eight babies in this study developed NNJ and of these, only 1 was HIV-positive - this was a statistically significant finding. The higher incidence of NNJ among the babies of HIV-negative women is in keeping with the findings by Nakanga et al. ${ }^{[24]}$ in a study undertaken in Malawi in 2015. They hypothesised that the efavirenz (EFV) in the prevention of mother-to-child transmission (PMTCT) of HIV acted as a fetal liver enzyme inducer that would aid the conjugation of bilirubin. All women in this study were on a triple ART regimen that included EFV. However, in contrast to this and other studies, the male sex was not a risk factor for neonatal jaundice in this study. ${ }^{[25,26]}$

The seropositivity rate in this study was $47 \%$, which was higher than the KZN provincial seropositivity rate of $40.1 \%$, but this may be due to the fact that HIV-positive women are more prone to sepsis and hence PPROM. ${ }^{[27]}$ Half of the women had CD 4 counts of $>350$ cells $/ \mu \mathrm{L}$, while $12 \%$ had CD 4 counts of $<200$ cells $/ \mu \mathrm{L}$. It is important to note that all the women were either on long-term or prophylactic ART, which consists of the fixed-dose combination of tenofovir (TDF), emtricitabine (FTC) and EFV as per national protocol. There was no association between low CD4 count and adverse outcomes, possibly because of the effect of the ART on suppressing the viral load. ${ }^{[28]}$

The mean number of antenatal visits and mean gestational age at booking were similar between the HIV-positive and HIV-negative groups. There was no obvious association between PPROM and maternal age, poor antenatal clinic attendance or parity in this study. High-risk maternal age groups, teenagers and women $>34$ years of age accounted for $26 \%$ of the women in this study. Nulliparous women constituted $33 \%$ of women with PPROM, which was lower than in previous similar studies. ${ }^{[29,30]}$
Seventeen percent of the women had the rupture of membranes confirmed by digital per vaginam examination despite the leakage of fluid having been noted in the patient history. Standard protocol recommends the use of a speculum to prevent ascending infection. ${ }^{[31]}$ We found no obvious explanation for this, however, disregard for protocol and the shortage of speculums were considered among the reasons for diagnosing PPROM by doing digital per vaginam examinations. In his study of 97 women with PROM in Chris Hani Baragwanath General Hospital (CHBGH), Iloanusi ${ }^{[30]}$ found that $35 \%$ of the women had a digital examination prior to the onset of labour, which was double the percentage compared with our study.

The most common microorganisms causing PPROM in this setting could not be determined due to the consistent omission of urogenital septic screening in nearly all of the women in this study. Iloanusi ${ }^{[30]}$ also found the same transgression at CHBGH. The lack of microbiological investigations did not seem to have any negative impact on the outcomes, as sepsis was not a significant finding in this study. In accordance with hospital protocol based on scientific evidence nearly all women were given prophylactic antibiotics (erythromycin and metronidazole). ${ }^{[32-34]}$ Babies delivered after prolonged rupture of membranes also received a course of intravenous antibiotics as standard neonatal protocol.

In resource-constrained areas, it could be argued that routine urogenital septic screening of patients with PPROM is not essential, particularly if immediate delivery is anticipated and if antibiotics are used routinely. Even if clinical chorioamnionitis is present, Osmanagaoglu and Unal ${ }^{[35]}$ found that neonatal outcomes in such patients were similar to those without clinical chorioamnionitis.

Following completion of steroids, a choice between expectant management and delivery is made. ${ }^{[36]}$ The protocol in our hospital setting recommends delivery of the neonate regardless of gestation because of concerns about the risk of infection and other complications associated with prolonged rupture of membranes. The fewer adverse outcomes in this study compared with others elsewhere seem to validate this school of thought.

The rate of CD in this study was 33\%, which was slightly higher than in similar studies by Iloanusi ${ }^{[30]}$ at $\mathrm{CHBGH}$ and by Patil and Patil ${ }^{[29]}$ in India, who reported $\mathrm{CD}$ rates of $25 \%$ and $27 \%$, respectively. The rate of $\mathrm{CD}$ for failed induction of labour was $11.1 \%$ compared with $7.6 \%$ in the Indian study and $16 \%$ in the CHBGH study.

\section{Study limitations}

Despite the limitations inherent to a retrospective study and the small sample size, this study provides a more objective comparison between HIV-positive and HIV-negative women with PPROM, given the homogeneity of the 2 groups in our study and the similarity in their management. Perhaps, more significantly, this study also reports some encouraging data on the benefit of the use of ART in all HIV-positive pregnant women regardless of their CD4 counts.

A major limitation of this study was that a significant number of files were either missing or were excluded due to a deficiency of crucial information required for the study, but this may not have had a negative impact on the overall findings because files from HIV-positive and HIV-negative women stood equal chances of being excluded. Deviation from the standard protocol of screening for infections in a form of midstream urine and vaginal swabs was noted. The extent to which sepsis is a risk factor for the PPROM and 
the most common pathogenic organism could not be established. The above two points are concerning in view of the rising litigation claims being made against the public and private sectors and the cost of settling such claims. ${ }^{[37]}$ If the effect of medical litigation in obstetrics is to be minimised, it is absolutely imperative that clinicians and nurses ensure that evidence-based practice guidelines are followed, that medical records are comprehensively completed and that administrative staff ensure that records are safely stored. ${ }^{[35]}$

\section{Conclusion}

This study suggests that there are no immediate significant differences in neonatal and maternal outcomes in pregnancies complicated by PPROM between HIV-negative and HIV-positive women who are on prophylactic or long-term ART, except in the development of NNJ, where ART was protective.

PPROM did not seem to significantly compound adverse perinatal outcomes associated with preterm delivery when the management strategy of immediate delivery after steroid fetal lung maturation, regardless of gestation, was employed.

A larger and more powered study is recommended to confirm the findings of this study. Furthermore, intermediate and longterm follow up is suggested to determine the development of late neonatal and maternal complications in this setting.

Acknowledgements. The authors would like to thank the administrative, nursing and medical staff who assisted with the study.

Author contributions. GS was the principal investigator and MN was the main research supervisor while $\mathrm{BH}$ provided specialist input. All authors were responsible for the drafting of the publication.

Funding. None.

Conflicts of interest. None.

1. French JI, McGregor JA. The pathobiology of premature rupture of membranes. Semin Perinatol 1996;20(5):344-368. https://doi.org/10.1016/s0146-0005(96)80002-4

2. Taylor J, Garite TJ. Premature rupture of membranes before fetal viability. Am J Obstet Gynecol 1984;64:615-620. http://dx.doi.org/10.1016/0002-9378(90)91080-V

3. Smith G, Rafuse C, Anand N, et al. Prevalence, management, and outcomes of preterm prelabour rupture of the membranes of women in Canada. J Obstet Gynaecol Can 2005;27(6):547-553. https:// rupture of the membranes of women in Can
doi.org/10.1016/s1701-2163(16)30711-3

4. Maymon E, Chaim W, Sheiner E, Mazor M. A review of randomised clinical trials of antibiotic therapy in preterm premature rupture of membranes. Arch Gynecol Obstet 1998;261(4):173-181 https://doi.org/10.1007/s004040050218

5. McGregor JA, French JI, Lawellin D, Todd JK. Preterm birth and infection: Pathogenic possibilities. Am J Reprod Immunol Microbiol 1988;16:123-132.

6. Simhan HN, Canava TP. Preterm premature rupture of membranes: diagnosis, evaluation and management strategies. Br J Obstet Gynaecol 2005;112:32-37.

7. Temmerman M, Chomba EN, Ndinya-Achola, Plummer FA, Coppens M, Piot P. Maternal human immunodeficiency virus-1 infection and pregnancy outcome. Obstet Gynecol 1994;83:495-501. https://doi.org/10.1097/00006250-199404000-00002w

8. Kumar RM, Uduman SA, Khurrana, AK. Impact of maternal HIV-1 infection on perinatal outcome. Int J Gynecol Obstet 1995;49(2):137-143. https://doi.org/10.1016/0020-7292(95)02356-h

9. Markson LE, Turner BJ, Houchens R, Silverman NS, Cosler L, Takyi BK. Association of maternal HIV infection with low birth weight. J Acquir Immune Defic Syndr Hum Retrovirol 1996;13:227234. https://doi.org/10.1097/00042560-199611010-00004
10. Langston C, Lewis DE, Hammill HA, Popek EJ, Kozinetz CA, Kline MW. Excess intrauterine fetal demise associated with maternal human immunodeficiency virus infection. J Infect Dis 1995;172:1451-1460. https://doi.org/10.1093/infdis/172.6.1451

11. Goldberg RL, Thompson C. The infectious origins of stillbirths. Am J Obstet Gynecol 2003;189(3):861-873. https://doi.org/10.1067/s0002-9378(03)00470-8

12. Martin R, Boyer P. Incidence of premature birth and neonatal respiratory disease in infants of HIVpositive mothers. J Pediatr 1997;131:851-856.

13. Kennedy D, Fawcus S, Kroon M. The effect of maternal HIV status on perinatal outcome at Mowbray Maternity Hospital and referring midwife obstetric units, Cape Town. SA J Obstet Gynaecol 2012;18(1):6-10. doi:10.7196/sajog.417

14. Pattison RC, Hulsbergen MH, van Hoorick L. The effects of maternal HIV infection on maternal condition and perinatal death in southwest Tshwane. Facts Views Vis ObGyn 2010;2(4):227-231.

15. De Knijf A, Pattison RC. Confidential enquiries into quality of care of women in labour using hypoxic ischaemic encephalopathy as a marker. Facts Views Vis ObGyn 2010;2(4):219-225.

16. Temmerman M, Nyongo AO, Bwayo J, Fransen K, Coppens M, Piot P. Risk factors for motherto-child transmission of human immunodeficiency virus-1 infection. AJOG 1995;172(2):700-705 https://doi.org/10.1016/0002-9378(95)90597-9

17. St Louis ME, Kamenga M, Browa C, et al. Risk of perinatal HIV-1 transmission according to maternal immunologic, virologic and placental factors. JAMA 1993;269(22):2853-2859. https://doi. org/10.1001/jama.1993.03500220039023

18. Kuhn L, Abrahams E, Matheson P, et al. Timing of maternal-infant HIV transmission: Association between intrapartum factors and early polymerase chain reaction results. AIDS 1997;11(4):429-435. https://doi.org/10.1097/00002030-199704000-00005

19. Garcia-Tejedor A, Perales A, Maiques V. Duration of rupture of membranes and extended labor are risk factors for HIV transmission. Int J Gynecol Obstet 2003;82(1):17-23. https://doi.org/10.1016/ s0020-7292(03)00123-1

20. Toumala RE, Shapiro DE, Mofenson LM, et al. Antiretroviral therapy during pregnancy and the risk of an adverse outcome. N Engl J Med 2002;346:1863-1870. https://doi.org/10.1056/nejmoa991159

21. Townsend CL, Cortina-Borja M, Peckham CS, Tookey PA. Antiretroviral therapy and premature delivery in diagnosed HIV infected women in the United Kingdom and Ireland. AIDS 2007;21(8):1019-1026. https://doi.org/10.1097/qad.0b013e328133884b

22. Braddick MR, Kreiss JK, Embree JE, et al. Impact of maternal HIV infection on obstetrical and early neonatal outcome. AIDS 1990;4(10):1001-1006. https://doi.org/10.1097/00002030-19901000000009

23. Lepage P, Dabis F, Hitimana DG, et al. Perinatal transmission of HIV-1: Lack of impact of maternal HIV infection on characteristics of livebirths and on neonatal mortality in Kigali, Rwanda. AIDS 1991;5(3):295-300. https://doi.org/10.1007/978-1-4615-2530-1_18

24. Nakanga W, Patel P, Panjwani S, Kennedy N, Kawaza K. Supra-treatment threshold neonatal jaundice: Incindence in HIV-exposed compared to non-exposed neonates at Queen Elizabeth Central Hospital in Blantyre, Malawi. Malawi Med J 2015;27(3):104-108. https://doi.org/10.4314/mmj.v27i3.7

25. Maisel MJ, Clifford K, Antle CE, Leib GR. Jaundice in the healthy newborn infant: A new approach to an old problem. Pediatrics 1988;81(4):505-511.

26. Najib KS, Saki F, Hemmati F. Incidence, risk factors and causes of sever neonatal hyperbilirubinemia in south of Iran (Fars province). Iran Red Crescent Med J 2013;15(3):260-263. https://doi. org/10.5812/ircmj. 3337

27. National Department of Health, South Africa. The 2013 National Antenatal Sentinel HIV Prevalence Survey South Africa. Pretoria: NDoH, 2015:27.

28. Geetha S, Ravindra P, Rashmi M. A prospective study of obstetric and newborn outcome in a cohort of HIV-affected pregnant women. JEMDS 2015;4:514-524. https://doi.org/10.14260/jemds/2015/77

29. Patil S, Patil V. Maternal and foetal outcome in premature rupture of membranes. IOSR JDMS 2014;13(12):56-83. https://doi.org/10.9790/0853-131275683

30. Iloanusi NE. Evaluation of pregnant women admitted with prelabour rupture of membranes. June 2013, http://wiredspace.wits.ac.za/jspui/bitstream/10539/13746/3/Iloanusi\%20MMed\%20Final\%20 Research\%20Report\%20Submission.pdf (accessed 4 June 2016).

31. Lewis DF, Major CA, Towers CV, et al. Effects of digital vaginal examination on latency period in preterm rupture of membranes. Obstet Gynecol 1992;80:630-634. https://doi.org/10.1016/00029378(91)91234-n

32. Kenyon S, Boulvain M, Neilson J. Antibiotics for preterm rupture of the membranes: A systematic review. Obstet Gynecol 2004;104(5 Part 1):1051-1057. https://doi.org/10.1097/01. aog.0000143268.36682.21

33. Mercer BM. Antibiotic Therapy for reduction of infant morbidity after preterm premature rupture of the membranes. JAMA 278(12):989-995. https://doi.org/10.1001/jama.1997.03550120049032

34. Osmanağaoğlu MA, Ünal S, Bozkaya $\mathrm{H}$. Chorioamnionitis risk and neonatal outcome in preterm premature rupture of membranes. Arch Gynecol Obstet 2004;271(1):33-39. https://doi.org/10.1007/ s00404-004-0644-8

35. Cox SM, Leveno KJ. Intentional delivery versus expectant management in premature rupture on membranes at 30 - 34 weeks gestation. Obstet Gynaecol 1995;875:879. https://doi.org/10.1016/0029 7844(95)00303-9

36. Bateman C. Medical negligence pay-outs soar by 132\% - subs follow. SAMJ 2011;101(4):216-218 https://wdoi.org/10.7196/samj.4881 